\title{
Probe Embryonic Damage Evolution in Bulk Metallic Glasses under Plate-impact Loading
}

\author{
Zhong Ling ${ }^{1, *}$, Xin Huang ${ }^{2}$, and Lanhong Dai ${ }^{1}$ \\ ${ }^{1}$ LNM, Institute of Mechanics, Chinese Academy of Sciences, Beijing 100190, China \\ ${ }^{2}$ ISE, China Academy of Engineering Physics, Mianyang 621999, P.R. China
}

\begin{abstract}
Microdamage in very short stress durations of spallation process in Zr-based bulk metallic glass (Zr-BMG) samples were captured by a specially designed plate impact technique. With stress durations vary, microdamage "frozen" in Zr-BMG samples exhibited different damage levels. Based on the morphology and stress environment of the microdamage, a compound microdamage evolution mode is applied to characterize the spallation evolution in Zr-BMGs. Especially the spallation in BMGs originates from cavitation instabilities in the weak regions with higher free volume content, which results in formation of ductile damage zones. The activation of shear transformation zones (STZs) or tension transformation zones (TTZs) between these ductile damage zones finally leads to detached spallation.
\end{abstract}

\section{Introduction}

Spallation is a dynamic failure phenomenon of solids under impulsive loadings, which is generated by interaction of release waves reflected from free surfaces [1]. In many areas such as military and aerospace, the consideration of spallation is of considerable importance in practical applications. Therefore, the mechanism of spallation has been extensively investigated over the past few decades [1-6]. In polycrystalline materials, it is well known that spallation is attributed to nucleation, growth and coalescence of micro-voids $[3,6]$ or micro-cracks $[1$, 4]. Whereas in bulk metallic glasses (BMGs) which are amorphous metastable solids with unique disordered atomic structures $[7,8]$, the mechanism of spallation is not yet clear. There have been two views to understand spallation in BMGs [9-12]. One suggests that the spallation is shear-induced brittle failure. As the zipshaped cracks or vain patterns are observed on crosssections or spall surfaces of recovered BMG samples in plate-impact experiments $[9,10]$, it is believed that the spallation results from formation and development of shear bands. Another view contends that the spallation is a mean tensile stress dominated cavitation process. For the voids or dimple structures are found in spalled samples $[11,12]$, it is implied that the spallation occurs via nucleation, growth and coalescence of micro-voids. Noticing that most of these observations are based on completely spalled samples, the real physical picture of spallation damages before detached spallation still remains unclear. Particularly, to uncover the origin of spallation in BMGs, the direct observation of microdamages at the early stage is needed. Recently, the early development of spallation damages in a Zr-based BMG, within duration of $10^{2} \mathrm{~ns}$ order, is probed by a "double- flyer" plate-impact experimental technique [13]. The clear pictures of early-stage spallation damages were observed on the recovered Zr-BMG samples after the plate-impact tests. And a damage evolution mode is proposed to understand the spallation mechanism in BMGs.

\section{Experimental procedure and results}

\subsection{Materials and experiments}

A typical BMG with the nominal composition $\mathrm{Zr}_{41.2} \mathrm{Ti}_{13.8} \mathrm{Cu}_{12.5} \mathrm{Ni}_{10} \mathrm{Be}_{22.5}$ (Vit.1) was adopt as the sample material in the plate-impact experiments. It was identified to be fully amorphous by conventional $\mathrm{x}$-ray diffraction (XRD). The flyer material was $\mathrm{H} 62$ brass (similar to $\mathrm{C} 28000$ brass, e.g., $62 \% \mathrm{Cu}, 38 \% \mathrm{Zn}$ ), which has nearly the same impedance as Vit. 1. The BMG samples were machined to disks of $20 \mathrm{~mm}$ in diameter and $\sim 2.5 \mathrm{~mm}$ in thickness, with both sides lapped and polished.

A "double-flyers" plate-impact experimental technique was applied to make stress durations short enough to avoid the occurrence of detached spallation. Using this technique, one can not only maintain a proper flyer thickness, but also control short stress pulses accurately [13]. Figure 1 shows the experimental configuration of the "double-flyers" technique for spallation experiments. Three BMG samples are targeted by the three sets of double-flyers with gaps of varied sizes, so that microdamage in different stress durations, under the same stress amplitude, could be "frozen" in the Zr-BMG samples. The details of "double-flyers" plate-

Corresponding author: lingz@lnm.imech.ac.cn 
impact experimental technique can be referred our previous work [13].

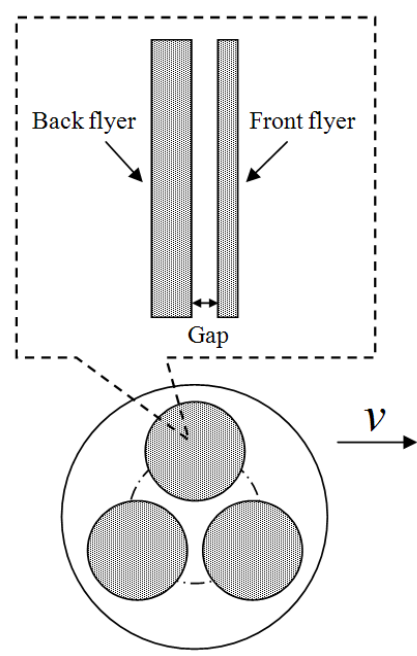

(a) Impactor

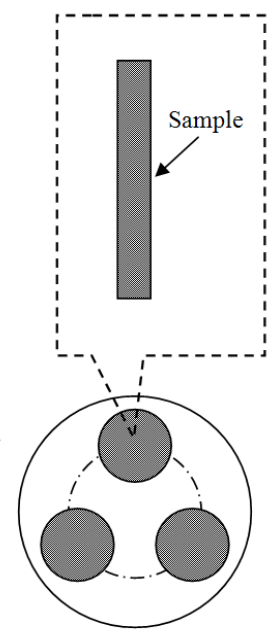

(b) Target
Fig. 1. Experimental configuration for "double flyer" spallation experiments (a) Impactor. (b) Target.

In each test, the impact velocity was kept at $200 \mathrm{~m} / \mathrm{s}$, corresponding to the stress amplitude of $3.2 \mathrm{GPa}$. The gap distances were controlled to be $25 \mu \mathrm{m}, 50 \mu \mathrm{m}$ and $75 \mu \mathrm{m}$, and the stress durations were $125 \mathrm{~ns}, 250 \mathrm{~ns}$ and 375 ns respectively. After the impact experiments were performed, samples were recovered. To observe the induced microdamage, each of recovered samples was cut along its diameter and then the cut-section was polished carefully. The polished faces were further electro-etched with a solution of $25 \%$ nitric acid in methanol below $0{ }^{\circ} \mathrm{C}$ at $\sim 10$ volts [14]. With the above procedure, small-scale voids and cracks can be clearly observed, and preferentially etched shear bands (if any) will appear. All the cross-sections were examined with scanning electron microscopy (SEM).

\subsection{Microscopic observations}

Detached spallation did not occur in all the tested samples, however, small-scale voids and microcracks appeared in the polished, electro-etched cross-sections of recovered samples.

Figure 2 is micrographs in low magnifications on partial cross-sections of the samples under the loading of $3.2 \mathrm{GPa}$. As shown in the figure, while the stress durations vary, the recovered samples exhibit strip-like concave regions at different levels. The strip-like concave regions, $10 \mu \mathrm{m}$ in length and parallel to each other, have been verified as spallation microdamage regions [14]. For the sample with the stress duration of $125 \mathrm{~ns}$ in Figure 2a, no spallation damage is found on the cross-section. As the stress duration increased to $250 \mathrm{~ns}$ in Figure 2b, two microdamage regions are seen near the pre-determined spall plane, where tensile stress gets its maximum. Figure $2 \mathrm{c}$ shows the sample of the duration of $375 \mathrm{~ns}$ with microdamage regions increasing.

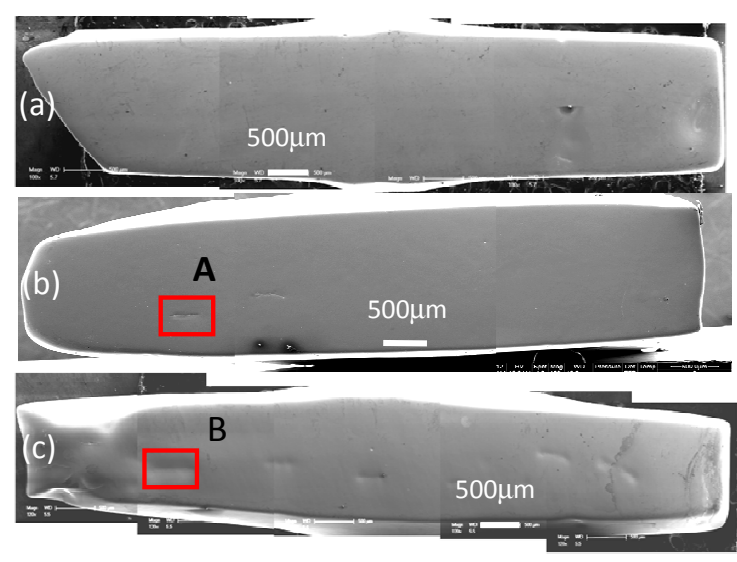

Fig. 2. Part of cross-sections of the recovered samples under stress amplitude of $3.2 \mathrm{GPa}$ and durations of (a) $125 \mathrm{~ns}$; (b) 250 ns and (c) $375 \mathrm{~ns}$.

To get more information of the spallation damages, the damage regions in the cross-sections of the samples were examined carefully. Figure 3 displays the damage zones in the samples with the stress duration of $250 \mathrm{~ns}$ and $375 \mathrm{~ns}$ (marked by A and B in Figures $2 \mathrm{~b}$ and $2 \mathrm{c}$ ). Figure $3 \mathrm{a}$ is an enlarged image of zone-A in Figure $2 \mathrm{a}$. The strip-liked microdamage region of $10^{2} \mu \mathrm{m}$ consists of several disconnected micro-flaws, appearing in predetermined spall plane. The longest flaw is about 150 $\mu \mathrm{m}$ and the others' length varies from 10 to $30 \mu \mathrm{m}$. Among them some penny-shaped cracks with length of $10 \mu \mathrm{m}$ appear [15]. Figure $3 \mathrm{~b}$ is an enlarged show of zone-C in Figure $3 \mathrm{a}$, where a part of the crack of $30 \mu \mathrm{m}$ is slightly curvilinear, inferring the long crack may be formed by coalescence of several short cracks [15]. Figure $3 c$ is a detail of zone-D (Figure $3 b$ ), in which equiaxial dimples of $10^{2} \mathrm{~nm}$ distributed in the crack visible surfaces. The dimple structure suggests that the cracks are formed by nucleation, growth and coalescence of nano- or micro-voids, thus this stage of microcrack evolution is ductile voids dominated. In other words, in this stage, short cracks such as penny-shaped cracks of $10 \mu \mathrm{m}$ [15], propagate as a result of ductile voids nucleation, growth and coalescence. And then some of the cracks link each other to form another longer straight one. Especially dimples without any distortion shown in Figure $3 \mathrm{c}$ also imply that all cracks, shorter or longer, are under hydro-static tensile stresses and located in a same plane, the predetermined spall plane.

Figure $3 d$ is an enlarged picture of region-B marked in Figure $2 b$, obtained from the sample of duration of 375 ns. As shown in the figure, micro-damage distributed in the sample looks like zigzag, no longer develops along one straight line as that in the sample of duration 250ns (Figure 3a). Details of microdamage zones in the sample, in higher magnifications, are presented in Figures $3 \mathrm{e}$ and $3 \mathrm{f}$, respectively. As exhibited in the figures, although there are a few voids with certain spacings (Figure 3e), relatively smooth fracture surfaces of zigzag-shaped cracks are observed between the voids (Figure 3f). Such zigzag morphology and relatively smooth fracture surfaces suggest that, within duration of $375 \mathrm{~ns}$, micro-cracks nucleation 
between two adjacent voids may occur via plastic strain localization.

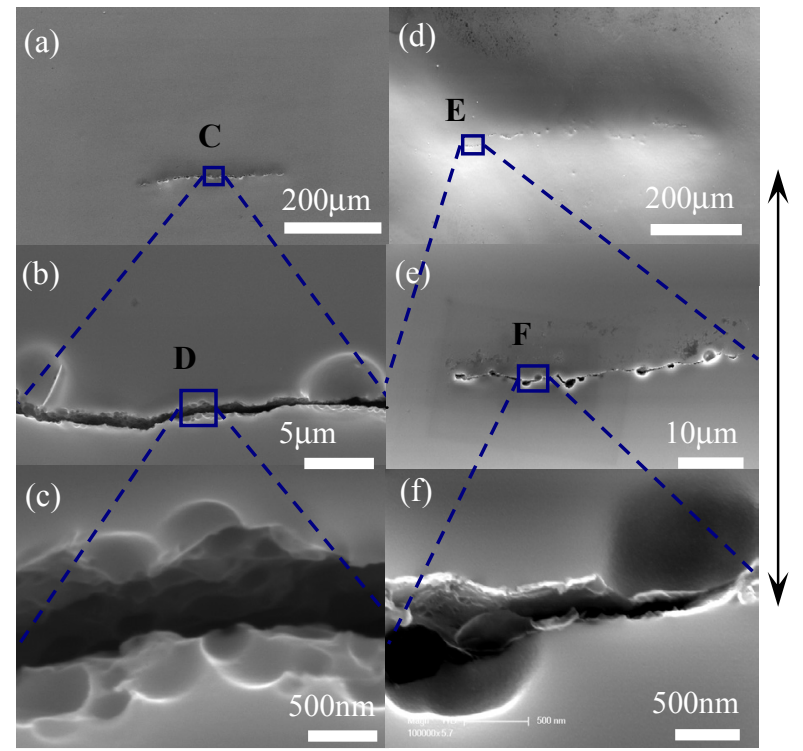

Fig. 3. Spallation damage zones on the cross-section of the samples, where the arrow denotes loading direction: (a) full-view of region-A in Figure $2 \mathrm{~b}$ (duration=250ns); (b) an enlarged show of zone-C; (c) the detail of zone-D; (d) full-view of region-B in Figure 2c (duration=375ns); (e) an enlarged show of zone-E; (f) the detail of zone-F.

\section{Microdamage evolution mode}

Different microdamage morphologies in current samples exhibit that microdamage evolution modes become different with stress duration varied. The strip-liked microcracks and dimples on their visible surfaces (Figures 3a, 3b and 3c) represent that microcracks are ductile-void dominated within duration $250 \mathrm{~ns}$. And the zigzag morphology and relatively smooth fracture surfaces (Figs 3d, 3e and 3f) indicate that brittle cracks nucleation is induced by shear localization with longer stress duration. Such evolution mode changing can be attributed to either stress environment or microstructure of the samples [1].

\subsection{Stress and strain environment}

Obviously, microcracks developing dominated by coalescence of micro-voids occurs under hydro-static tensile stresses and on the same predetermined spall plane. And the zigzag microdamage showed in Figures $3 \mathrm{~d}$ and $3 \mathrm{e}$ is attributed to developing or linking between two adjacent interlaced micro-cracks local at two parallel planes. Especially interlaced microcracks' appearing is dependent on their stress and strain environments.

As we known, in solids, spallation microdamage nucleates at the locations where composing of stress amplitudes and durations gets the microdamage nucleation criteria of the materials [1]. That is, the microdamage nucleates under a given stress amplitude within a stress duration. In earlier stage, microcracks appear first in the predetermined spall plane, for composing of the maximum amplitude and the shortest duration of stress reaches at the criteria of the BMGs.

Once microdamage is nucleated, new microdamage does not appear in regions adjacent the old one due to stress-relaxation. Thus disconnected microcracks appear along a strip, as shown in Figures $3 \mathrm{a}$ and $3 \mathrm{~b}$. In this case, microcracks, including those formed by two adjacent microcracks' linking each other, are all in the same plane [15].

Whereas with stress duration getting longer, when composing of the amplitude and duration of the stress in some new sites reaches at the nucleation criteria, other new microcracks may appear. The new microcracks nucleate usually slight later than that old ones, so their sites may be a little far away from the predetermined spall plane. Due to stress-relaxation also in this case, the new microcracks had to appear interlaced with the old ones. Henceforth with stress duration longer, two adjacent interlaced micro-cracks located at two planes are linked in precedence, leading to zigzag morphologies of microdamage (Figures $3 \mathrm{~d}$ and $3 \mathrm{e}$ ). Moreover, the cracks' linking between two adjacent microcracks in two planes is of course dominated by deviator stresses. This is quite different from that of ductile-void dominated microcracks which are governed by hydrostatic tensile stress only.

\subsection{Microstructure heterogeneity}

As demonstrated in previous studies, micro-structure heterogeneity is another key factor which would lead to ductile void growth and brittle crack propagation in solids [1]. In polycrystalline solids microstructure heterogeneities are grains and grain boundaries where dislocations pile-up. While micro-voids is nucleated in grain boundaries, ductile void growth and brittle crack propagation can occur intergranularly (in the grain boundaries) or transgranularly (inside the grains) [1].

However, in contrast to those of polycrystalline solids, microstructure of BMGs is a unique amorphous structure or disorder atomic structure. Due to no grains and grain boundaries, BMGs seem to be homogeneous in macroscopic scales like glasses. Thus microstructure heterogeneities that may affect microdamage evolution mode changing in BMGs, merely come from free volumes, which are the intrinsic defects of the materials. Actually mechanical properties of BMGs [16-18] are very sensitive to contents of free volume and, with the change of free volume contents BMGs can exhibit different behaviour [19]. In addition, contents of free volumes are dependent on stress environment of the BMGs [20, 21], i.e. high contents of free volumes always exist in there where hydro-static tensile stress is, such as shear bands [20] or spallation microdamage plane [12] as well as the predetermined spall plane in current samples. As exhibited in Figures $2 b$ and $2 c$, it is the high contents of free volumes in predetermined spall plane that supply possibilities for microdamage nucleation in the plane. Nevertheless microdamages shown in Figures $2 b$ and $2 c$ do not occur anywhere along the plane. This illustrates that free volume is not well- 
distributed or uniform in BMGs even if in the places with high contents of free volumes.

\subsection{Compound damage evolution mode}

Based on the experimental observations and analysis above on stress environments, microstructure heterogeneities related to free volume in BMGs, a compound damage mode is proposed to understand the damage evolution process of spallation in the material. The compound damage evolution mode consists of two stages: the early stage for forming ductile damage zones and the late stage for linking the damage zones to cause detaching in larger scales. Besides the compound damage evolution mode can be understood as being progressively performed with scales varying from microscale to meso-scale and finally to form macroscopic spallation.

Figure 4 shows the early stage of spallation in BMGs. As the as-received BMG samples are not very homogeneous, the intact material of Zr-BMG samples contains weak and strong regions, depending on the free volume contents in the material [19]. Weak regions with a higher free volume content tend to exhibit a ductile behaviour (Figure 4a). Once the sample is undergone tensile loadings (Figure 4b), micro-damages are prone to nucleate and develop in weak regions leading to formation of ductile damage zones in predetermined spall plane where the maximum tensile stress achieves. The longer the stress duration is, the more weak regions are fractured.

To understand how these ductile damage zones originate from shear bands or micro-voids, molecular dynamics (MD) simulations are performed [22]. The simulations are performed on a simple binary metallic glass $\mathrm{Cu}_{50} \mathrm{Zr}_{50}$. The Finnis-Sinclair type potential with parameters given by Mendelev et al. [23] is adopted to describe the atomic interactions. A small glass sample with dimensions of $\sim 20 \mathrm{~nm} \times 20 \mathrm{~nm} \times 20 \mathrm{~nm}$ is prepared from a melting and quenching process. Then it is replicated along $x$ direction to obtain larger systems [24]. The final dimensions of flyers are $\sim 100 \mathrm{~nm} \times 20$ $\mathrm{nm} \times 20 \mathrm{~nm}$, while that of targets are $\sim 200 \mathrm{~nm} \times 20 \mathrm{~nm}$ $\times 20 \mathrm{~nm}$. Periodic boundary conditions are applied along the $y$ and $z$ directions to ensure a uniaxial strain state. As the flyer impacts the target at a velocity $V$, shock loading is generated and spallation damages will appear in the middle region of the target.

The spallation damage evolution process under planar shock wave loading $(V=1800 \mathrm{~m} / \mathrm{s})$ is presented in Figure 4c. As shown in the figure, cavitation instabilities $[25,26]$ occur clearly at the early stage of spallation, while no shear bands (bands with large nonaffine deformation [27]) are observed. During the cavitation process, tension transformation zones (TTZs) $[11,28]$, which are fundamental unit processes of collective atomic motion under tensile pressures, are arisen first to lead to nucleation of these cavities. Then shear transformation zones (STZs) [29] are activated by deviator stress around and between the cavities, resulting in cavities to grow and coalesce. Finally, ductile cracks are formed and void/dimple structures are left on the spall surface. This is the early stage when the

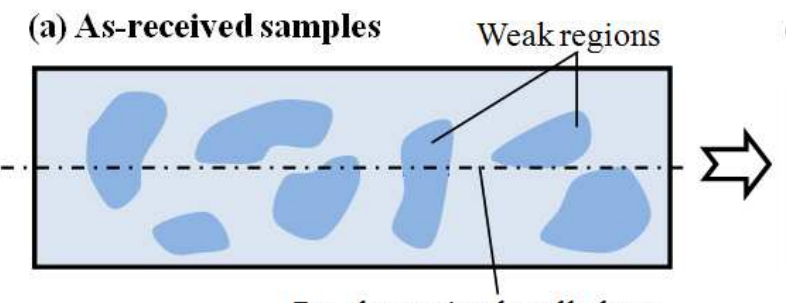

Pre-determined spall plane

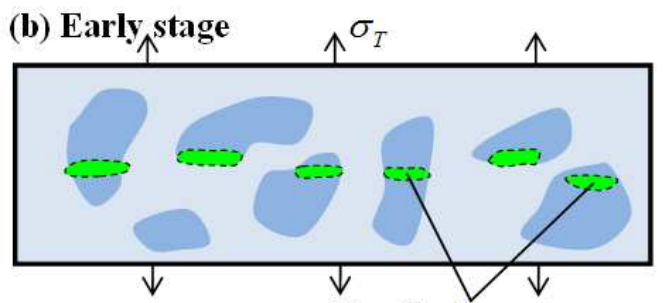

Ductile damage zones

(c) Cavitation instabilities in ductile damage zones

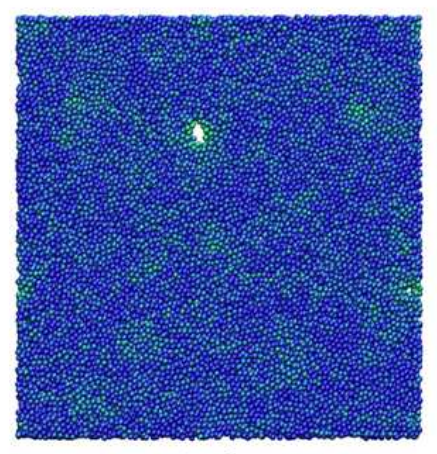

$\Delta \mathrm{t}=3 \mathrm{ps}$

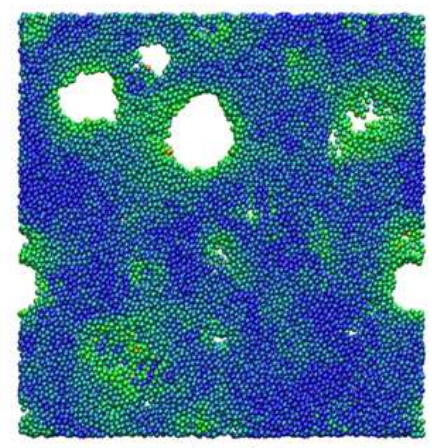

$\Delta \mathrm{t}=6 \mathrm{ps}$

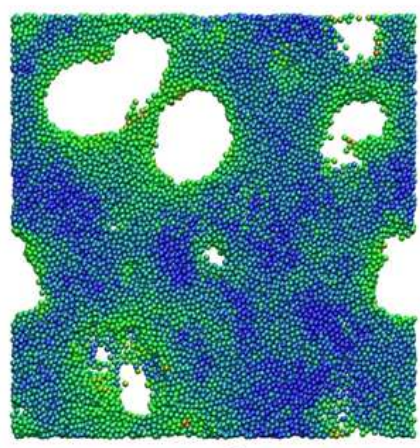

$\Delta \mathrm{t}=9 \mathrm{ps}$

Fig. 4. The early stage for forming ductile damage zones. (a) As-received samples containing weak regions. (b) Ductile damage zones formed in weak regions. (c) Atom-scale pictures showing cavitation instabilities in ductile damage zones. Molecules are colored according to the value of $D_{\min }^{2}$ over the time $\Delta \mathrm{t}$ from the same initial configuration, and the green represents higher non affine deformation. 
microdamage evolution of BMG samples exhibits a ductile behavior.

Figure 5 illustrates the late stage of spallation in
Microdamage evolution occurred in earlier stages of spallation in a Zr-BMG under uniaxial strain or hydrostatic tension has been analyzed. According to the (a) Samples with ductile damage zones

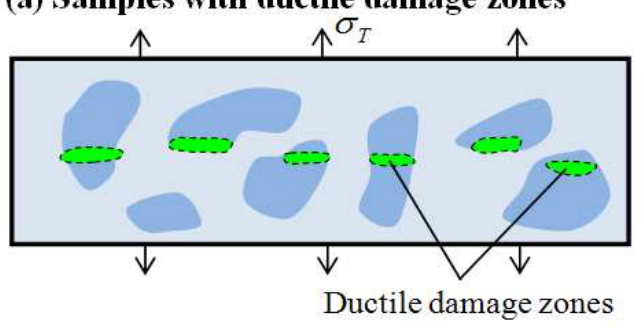

(c) Activation of TTZs and STZs

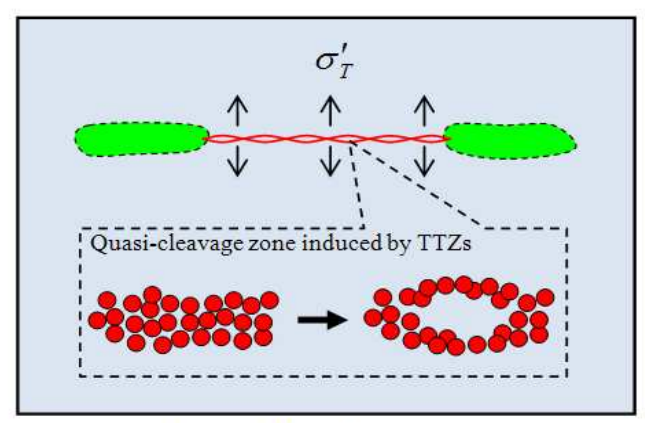

Cleavage flaws formation
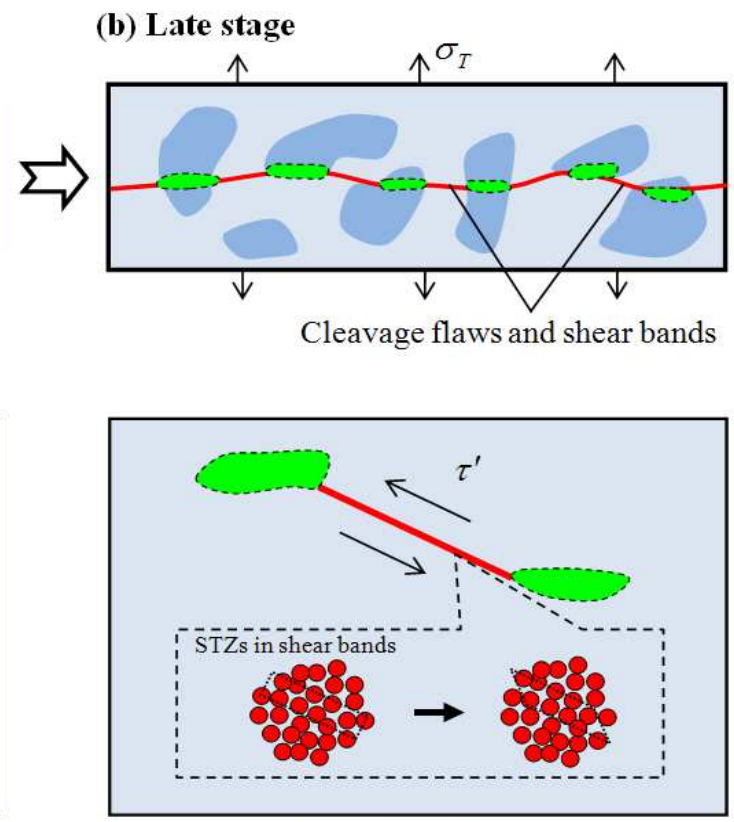

Shear bands formation

Fig. 5. The late stage for linking the ductile damage zones around macroscopic spall plane. (a) Samples with ductile damage zones. (b) Brittle damage formed in strong regions. (c) Activation of TTZs and STZs in strong regions.

BMGs. As analyzed above, with a longer duration, more weak regions are damaged around the predetermined spall plane so that damage zones are interlaced. In this case strong regions between ductile damage zones are undergone to stress concentrations, causing two adjacent microcracks' linking in the strong region. Thus brittle damages will form and develop (Figure 5b). Obviously, the ductile damage zones are randomly distributed around or deviated to the pre-determined spall plane, leading to different paths and stress states between ductile damage zones (Figure 5c). For two adjacent micro-cracks in the same plane in the ductile damage zones, cracks propagate fast into the strong regions from the ductile regions dominated by the hydro-static tensile stress. In this case, TTZs are activated near the crack tips and induce brittle cleavage of the material $[11,28]$. But for interlaced microcracks in the neighboring regions on different planes, deviator stresses or shear stresses dominate cracks propagating in the strong region between two ductile regions, therefore it is deviator stress that promotes the activation of STZs [29]. Then shear bands [30-32] will initiate and propagate, giving rise to failure of the strong region. This causes zigzag cracks and vain patterns. Furthermore, with all the strong regions are fractured, detached spallation occurs. That is why in the late stage the BMG samples exhibit a brittle behaviour.

\section{Conclusions}

morphology and stress environment of the microcracks in Zr-BMG samples, a compound damage evolution mode is proposed to characterize and understand the spallation mechanism in BMGs. The followings are deduced:

1. Microdamage evolution in very short stress durations were controlled accurately by a specially designed plateimpact technique. And with stress durations vary, microdamage "frozen" in Zr-BMG samples exhibited different damage levels;

2. The intact BMGs are regarded as being consisted of weak regions and strong regions, corresponding to higher and lower contents of free volume. In weak regions, microdamage is nucleated by coalescence of free volume and the nucleating sites are dependent on their stress environment. Thereupon a compound damage evolution mode consisting of the early and late stages is proposed;

3. The early stage is formation of ductile damage zones therefore it is ductile voids dominated. Further analysis via MD simulations revealed that, in this stage, microdamage zones are originated from cavitation instabilities in the weak regions, where tension transformation zones (TTZs) are arisen first under hydrostatic tensile stress to lead to nucleation of these cavities;

4. The late stage is linking of the damage zones to cause detaching in larger scales. It is brittle crack propagation in strong regions with lower contents of free volume. During the propagation, activation of shear transformation zones (STZs) or tension transformation 
zones (TTZs) between the ductile damage zones finally leads to the detached in larger scales.

Financial support was from the NSFC (Grants Nos.: 11772313, 11472287, 11402245, and11272328), the Key Research Program of Frontier Sciences (Grant No. QYZDJSSWJSC011), the Strategic Priority Research Program of the Chinese Academy of Sciences (Grant No. XDB22040302, XDB22040303 )

\section{References}

1. D.R.Curran, L. Seaman and D.A. Shockey, Phys. Rep., 147 5-6 253 (1987)

2. M.A. Meyers, Dynamic Behaviour of Materials (Wiley-Interscience, New York 1994)

3. J.N. Johnson, J. Appl. Phys., 522812 (1981)

4. Y. L. Bai, Z. Ling, L.M. Luo and F.J. Ke, J. Appl. Mech.-T. ASME, 59622 (1992)

5. R.J. Clifton, Int. J. Solids Struct., 37105 (2000)

6. A. Molinari and T.W. Wright, J. Mech. Phys. Solids, 531476 (2005)

7. M.W. Chen, Annu. Rev. Mater. Res., 38445 (2008)

8. M.L. Falk and J.S. Langer, Annu. Rev. Condens. Matter Phys., 2353 (2011)

9. S.M. Zhuang, J. Lu and G.. Ravichandran, Appl. Phys. Lett., 804522 (2002)

10. S.J. Turneaure, S.K. Dwivedi and Y.M. Gupta, J. Appl. Phys., 101043514 (2007)

11. J.P. Escobedo and Y.M. Gupta, J. Appl. Phys., 107 123502 (2010)

12. X. Huang, Z. Ling, H.S. Zhang, J. Ma and L.H. Dai, J. Appl. Phys., 110 (2011) 103519

13. Z. Ling, X. Huang and L.H. Dai, Hopkinson Centenary Conference Cambridge 2014, Cambridge, Stefan Hiermaier (ed.), 211-24 (2014)
C.A. Pampillo, Scripta Metall., 6915 (1972)

14. Z. Ling, X. Huang and L.H. Dai, DYMAT2015, Europe Physics Journal (EPJ) Web Conferences 94 02016 (2015)

15. T.W. Wu and F. Spaepen, Philos. Mag. B, 61739 (1990)

16. J.J.Lewandowski, W.H. Wang andA.L. Greer, Philos. Mag. Lett., 8577 (2005)

17. R. Raghavan, P. Murali P and U. Ramamurty, Acta Mater., 573332 (2009)

18. F. Spaepen, Acta Metall., 25407 (1977)

19. J. Li, F. Spaepen and T.C. Hufnagel, Philos. Mag. A, 822623 (2002)

20. K.M. Flores and R.H. Dauskardt, Acta Mater., 49 2527 (2001)

21. X. Huang, Doctoral dissertation, University of CAS (2013)

22. M.I. Mendelev, D.J. Sordelet and M.J. Kramer, J. Appl. Phys., 102043501 (2007)

23. B. Arman B, S.-N. Luo, T.C.Germann and T. Çağın, Phys. Rev. B, 81144201 (2010)

24. E. Bouchaud, D.Boivin, J.L.Pouchou, D.Bonamy, B.Poon and G..Ravichandran, Europhys. Lett., 83 66006 (2008)

25. E. Bouchbinder, T. S.Lo and I. Procaccia, Phys. Rev. E, 77025101 (2008)

26. M.L. Falk and J.S. Langer, Phys. Rev. E, 577192 (1998)

27. M.Q. Jiang, Z. Ling, J.X. Meng and L.H.Dai, Philos. Mag., 88407 (2008)

28. A.S. Argon, Acta Metall., 2747 (1979)

29. M.Q. Jiang and L.H. Dai, J. Mech. Phys. Solids, 57 1267 (2009)

30. L.H.Dai and Y.L. Bai, Int. J. Impact Eng., 35704 (2008)

31. Y.F. Gao, L. Wang, H. Bei and T.G. Nieh, Acta Mater., 594159 (2011) 